\title{
Cellular immunodeficiency related to chronic dermatophytosis in a patient with Schistosoma mansoni infection: can schistosomiasis induce immunodeficiency?
}

\author{
Maurício Domingues Ferreira ${ }^{[1]}$, Anna Cristina Collaniere ${ }^{[1]}$, Dalton Luís Bertolini ${ }^{[1]}$, \\ Noac Chuffi Barros ${ }^{[1]}$ and Dewton de Moraes Vasconcelos ${ }^{[1]}$
}

[1]. Ambulatório de Manifestações Cutâneas das Imunodeficiências Primárias, Departamento de Dermatologia, Hospital das Clínicas, Faculdade de Medicina, Universidade de São Paulo, São Paulo, SP, Brasil.

\begin{abstract}
:
Here, we describe a case of hepatosplenic schistosomiasis that progressed to widespread persistent dermatophytosis. Significant $\mathrm{T}$ and B lymphocytopenia was confirmed. T-cell deficit is associated with increased susceptibility to fungal infections of skin and mucous membranes. The accumulation of a large amount of blood cells in the spleen could have played a crucial role in the development of lymphocytopenia in the present case. Alternatively, the schistosomiasis-induced increase in prostaglandin E2 levels could have inhibited the production of interferon $-\gamma$, a cytokine fundamental to fungal resistance. This case shows the potential of hepatosplenic schistosomiasis to impair the immune response.
\end{abstract}

Keywords: Schistosomiasis. Immunodeficiency. Chronic dermatophytosis.

\section{INTRODUCTION}

Schistosomiasis is a tropical disease caused by the flatworm Schistosoma mansoni. Specific freshwater snails (Biomphalaria sp) act as intermediate hosts, and human contact with water contaminated with larvae (cercariae) leads to infection. Immunopathological reactions against Schistosoma eggs trapped in tissues lead to inflammatory and intestinal obstructive disease, hepatosplenic inflammation, and liver fibrosis. Portal hypertension leads ultimately to hypersplenism and cytopenias, mainly thrombocytopenia and neutropenia. Schistosomiasis affects more than 200 million people worldwide: 120 million are symptomatic and 20 million have severe disease ${ }^{1}$. Despite the severity and impact of schistosomiasis on public health, the literature contains no reports relating schistosomiasis to any form of immunodeficiency. Here, we report the case of a patient with hepatosplenic schistosomiasis who developed lymphocytopenia associated with chronic dermatophytosis, indicating secondary immunodeficiency.

\section{CASE REPORT}

A 37-year-old man born in the rural area of the State of Minas Gerais, Brazil, to non-consanguineous parents of Caucasian

Corresponding author: Dr. Maurício Domingues Ferreira

e-mail:madofe@uol.com.br

Received 24 May 2016

Accepted 8 September 2016 and African ascendancy, showed normal neuropsychomotor development and no major diseases up to the age of 21 years. At this age, extensive and chronic dermatophytosis developed on his trunk, legs, and feet, and in the gluteal, inguinal, and perineal regions (Figure 1).

There was multiple circular, oval, and circinate lesions, confluent with scaly and hypochromic centers, with a $1-\mathrm{cm}$ average diameter. A large, elevated, and erythematous lesion with a scaly center lesion that covered almost the entire upper back was particularly suggestive of a dermatophytosis. These lesions occurred intermittently, but 6 years prior, when the patient was 31 years old, the lesions became persistent, more extensive, and refractory to topical treatments. Abdominal enlargement occurred 4 years prior. His visited the Infectious Diseases Clinic, where a hepatosplenic form of Schistosoma mansoni infection was diagnosed. Treatment was initiated, and because of the uncommon pattern of the dermatophytosis, he was referred to the Primary Immunodeficiency Outpatient Unit for investigation of possible immunodeficiency. The patient had no history of sinusitis, pneumonia, cutaneous infection, meningitis, mucocutaneous or systemic candidiasis, chronic diarrhea, or other infections. His birthplace is endemic for S. mansoni. He had two brothers, one of whom died because of complications of $S$. mansoni infection, while the other is healthy. His uncle was also diagnosed with hepatosplenic schistosomiasis. During our initial investigation, dermatophytosis was confirmed by direct exam of the scraped skin from three different regions, which indicated the presence of multiple branched and septate 

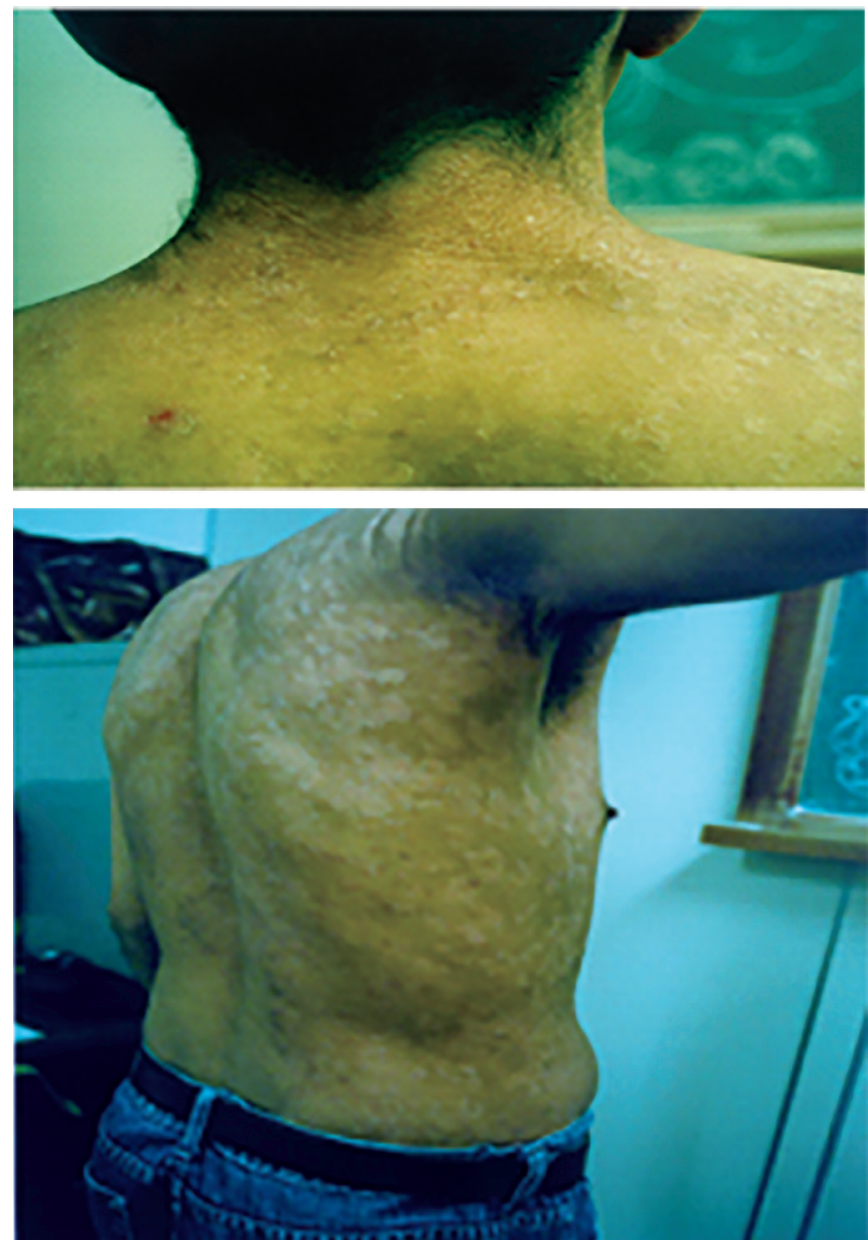

FIGURE 1. Photographs of the patient showing disseminated dermatophytosis due to Trichophyton rubrum. He presents typical and atypical lesions of dermatophytosis. These atypical lesions may be associated with a failure of the immune system to control the fungal infection.

hyaline hyphae, and macroconidia like small droplets located in parallel with the hyphae. Fungal cultures incubated for 4 weeks at $25^{\circ} \mathrm{C}$ on BD Mycosel Agar and BD Sabouraud Agar with chloramphenicol and cycloheximide (BD Biosciences, Franklin Lakes, NJ) revealed a dermatophyte with the morphology of Trichophyton rubrum, showing cotton wool-like filamentous colonies that had a raised center (obverse) and red pigmentation in the reverse view. Abdominal ultrasonography revealed hepatosplenomegaly. The liver showed characteristics of chronic hepatic disease with portal hypertension, and consequent splenomegaly (volume $=120 \mathrm{~cm}^{3}$; normal value $=20 \mathrm{~cm}^{3}$ ). Abdominal computed tomography confirmed the ultrasonography results (Figure 2).

Upper digestive endoscopy showed esophageal varices and erosive pangastritis. A myelogram showed normal myeloid series without morphological changes, normal maturation, and $8 \%$ eosinophils (possibly due to schistosomiasis), and normal erythropoiesis (with relative hypocellularity and low iron deposits); megakaryocytic series and lymphoplasmacytic series evidenced normal characteristics. The complete blood count showed mild sideropenic anemia without evidence of hemolysis, thrombocytopenia $\left(20,000\right.$ platelets $\left./ \mathrm{mm}^{3}\right)$, or leukopenia $\left(1,007\right.$ leucocytes $/ \mathrm{mm}^{3}, 600$ neutrophils $/ \mathrm{mm}^{3}$, and 400 lymphocytes $/ \mathrm{mm}^{3}$ ). Immunological tests revealed no deficiency in the humoral response. Immunoglobulin levels were normal, Immunoglobulin $\mathrm{G}$ ( $\mathrm{IgG}$ ) against measles were present, and $\mathrm{IgG}$ against toxoplasmosis, cytomegalovirus, hepatitis C virus, HBsAg (surface antigen of the hepatitis B virus), and human immunodeficiency virus were absent. Results of laboratory tests performed in July 2005 were as follows: leucocytes, $900 / \mathrm{mm}^{3}$; lymphocytes, $376 / \mathrm{mm}^{3}$; CD3+, 265/ $\mathrm{mm}^{3}$; $\mathrm{CD} 4+, 144 / \mathrm{mm}^{3}$; $\mathrm{CD} 8+112 / \mathrm{mm}^{3}$; CD4+/CD8+ ratio, 1.28 ;

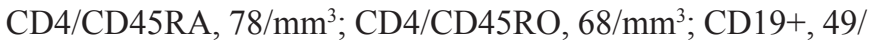
$\mathrm{mm}^{3}$; CD3-/CD56+, 38/ $\mathrm{mm}^{3}$; and CD3+/CD56+, 23/ $/ \mathrm{mm}^{3}$. The patient showed a normal lymphoproliferative response to the $\mathrm{T}$ and B mitogens PHA and OKT3, and PWM respectively, as well as to cytomegalovirus antigens, but no response to Candida and tetanus toxoid antigens. Since this time, he has presented with $\mathrm{T}$ - and B-cell lymphocytopenia. A second lymphoproliferation assay demonstrated a positive response to Candida antigen. The negative response to tetanus toxoid was sustained, and is probably related to delayed administration of the tetanus vaccine booster.

\section{DIsCusSION}

Around $5-7 \%$ of patients with hepatosplenic schistosomiasis progress to the severe form associated with periportal fibrosis, portal hypertension, splenomegaly, upper digestive tract bleeding and cytopenia ${ }^{2}$. These patients can experience hyperfibrinolysis, which may predispose them to gastrointestinal bleeding $^{3}$. Our patient had the hepatosplenic form of S. mansoni infection, with portal hypertension and huge splenomegaly, as well as neutropenia, thrombocytopenia and mild anemia, which indicated hypersplenism. Hypersplenism might explain the lymphopenia and immune defects observed, but was not consistent with the bone marrow biopsy results, which indicated erythropoiesis with relative hypocellularity and iron deficiency signs, as opposed to the hypercellularity expected in hypersplenism. Moreover, we found no evidence of hemolytic anemia, which is caused by the excessive destruction of red blood cells in the spleen in hypersplenism. The patient's anemia was likely provoked by chronic, imperceptible blood losses from esophageal varices, but this does not explain the thrombocytopenia and leukopenia. The myelogram showed normal counts and morphological features in all series (lymphoplasmacytic, myeloid, erythroid, and megakaryocytic), ruling out cytopenia-causing hematological diseases such as marrow aplasia, blood dysplasias, and hematologic neoplasias. In fact, the total number of lymphocytes in the bone marrow was normal, and we believe that the storage of a large number of blood cells, including platelets and leukocytes, in the spleen results in decreased numbers of these cells in the blood, which could lead to decreased number of peripheral lymphocytes. This abnormality is known to occur in this disease, but chronic fungal infections have never been observed. During an infection, defense cells are mobilized from the spleen to the periphery, triggering an immune response. The clinical features of the 


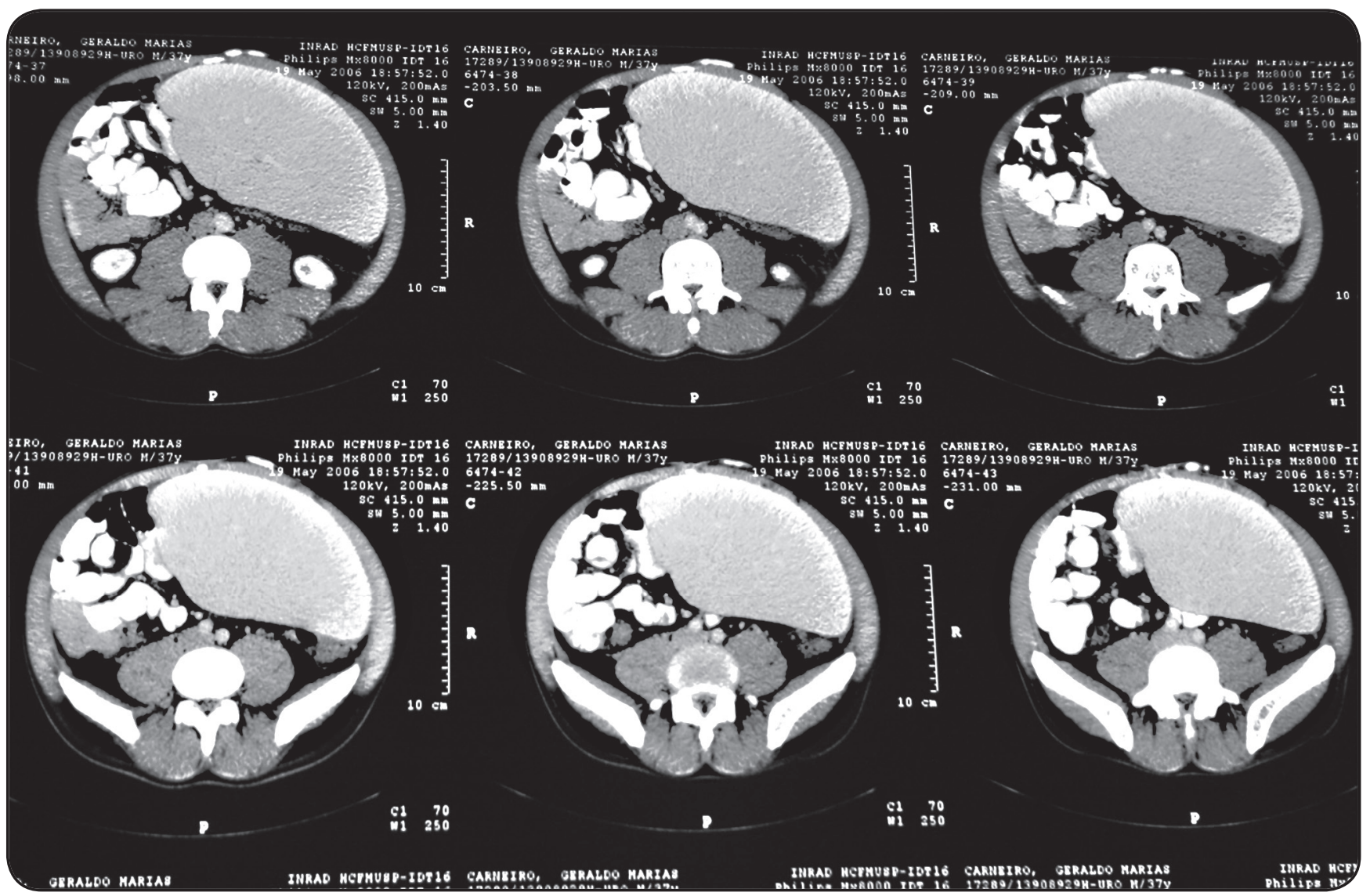

FIGURE 2. Computerized axial tomography showing the huge spleen in the iliac fossae. The patient's lymphocytopenia could be related to the storage of blood cells in this organ.

present case suggest inadequate functioning of this mechanism in our patient. Extensive persistent dermatophytosis reflects an inability to clear cutaneous fungal infections, strongly suggesting immune dysfunction. Most cutaneous lesions provoked by T. rubrum did not show the typical characteristics of this infection, a feature that might be associated with immunodeficiency. This raises the intriguing question of whether $S$, mansoni infection leads to immunodeficiency. Strong evidence supports the crucial role of the cellular immune response in the resistance to cutaneous fungal infection, and $\mathrm{CD} 3+\mathrm{T}$ lymphocytes play a central role in resistance to candidiasis ${ }^{4}$ and dermatophytosis 5 . Our patient presented with lymphocytopenia involving $\mathrm{T}$ $(\mathrm{CD} 3+), \mathrm{B}(\mathrm{CD} 19+)$, and natural killer cells $(\mathrm{CD} 3-/ \mathrm{CD} 56+)$, which likely impaired the immune response against cutaneous fungi, increasing his susceptibility to dermatophytosis. This susceptibility appears to be specific because no other infections were observed.

The increase in prostaglandin E2 (PGE2) levels in schistosomiasis could also be related to fungal susceptibility. Schistosomiasis is characterized by chronic granulomatous inflammation mediated by lymphocytes and soluble factors, such as cytokines, suppressor T cells, macrophages, and immune complexes (IC), which regulate the immune response during progression from acute disease to a chronic state. The precise role of these IC in immunological regulation remains unclear. However, the formation of granulomas against helminths egg antigens is likely related to the release of inhibitory mediators such as PGE2 by monocytes and macrophages ${ }^{6}$. Our previous study revealed that patients affected by extensive dermatophytosis caused by T. rubrum showed normal IL-12 and IL-10 expression, but decreased interferon-gamma (IFN- $\gamma$ ) release in response to in vitro immunogenic stimulation by T. rubrum peptides. . Moreover, T. rubrum extract did not stimulate the T cells of some healthy and affected patients, which could be related to mannans in the Trichophyton spp. extract ${ }^{5}$, which have an inhibitory effect on lymphocytes and their cytokine production ${ }^{11}$. In contrast, lymphocytes stimulated by tetanus toxoid or T. rubrum peptides in the control group produced IFN- $\gamma$. The mechanism of fungal inhibition of specific IFN- $\gamma$ production remains unknown, but intracellular factors within the host may be involved, since antigen-presenting cells internalize the fungus when they begin to release PGE2 ${ }^{7}$, and inhibit IFN- $\gamma$ production without modifying IL-4 release ${ }^{8}$. The most abundant prostaglandin in the human body, PGE2, can have both inflammatory and anti-inflammatory effects 9 . Kanda et al. ${ }^{10}$ showed that in patients with atopic dermatitis challenged by Candida albicans, inhibition of IFN- $\gamma$ release is associated with an increase in PGE2. Cytokine secretion was restored upon 
stimulation by T. rubrum extracts, which decreased PGE2 levels. In the present case, despite his moderate $\mathrm{B}$ cell lymphocytopenia (CD19+ cells), the patient's humoral response remained unaltered. His lymphoproliferative responses to mitogens and antigens were normal, except for the absence of a response to tetanus toxoid and Candida. This impaired response to Candida antigens reflects his inability to clear fungus appropriately, a function mediated by the cellular immune branch, even though he had never experienced an episode of candidiasis. Given the lack of evidence of hypersplenism and the high risk of performing a splenectomy, we decided not to perform surgery. At 2-years follow-up, the low levels of lymphocytes, neutrophils, and platelets persist, but without any intercurrence, and the fungal infection is controlled by intensive treatment with oral and topical azole antifungals.

\section{Conflict of interests}

The authors declare that there is no conflict of interest.

\section{REFERENCES}

1. Ross AGP, Bartley PB, Sleigh AC, Olds GR., Li Y, Williams GM, et al. Schistosomiasis. N Engl J Med. 2002;346(16):1212-20.

2. Leite LA, Domingues AL, Lopes EP, Ferreira RC, Pimenta Filho AA, da Fonseca CS, et al. Relationship between splenomegaly and hematologic findings in patients with hepatosplenic schistosomiasis. Rev Bras Hematol Hemoter. 2013;35(5):332-6.

3. Leite LA, Pimenta Filho AA, Martins da Fonseca CS, Santana dos Santos B, Ferreira RC, Montenegro SM, et al. Hemostatic dysfunction is increased in patients with hepatosplenic schistosomiasis mansoni and advanced periportal fibrosis. PLoS Neg1 Trop Dis. 2013; 7:e2314. doi: 10.1371/journal.pntd.0002314.

4. Fidel Jr PL. Immunity to Candida. Oral Dis 2002; 8 (suppl 2):69-75.

5. Bressani VO, Santi TN, Domingues-Ferreira M, Almeida A, Duarte AJ, Moraes-Vasconcelos D. Characterization of the cellular immunity in patients presenting extensive dermatophytoses due to Trichophyton rubrum. Mycoses. 2013;56(3):281-8.

6. Neves SM, Rezende SA, Goes AM. Nitric oxide-mediated immune complex-induced prostaglandin E (2) production by peripheral blood mononuclear cells of humans infected with Schistosoma mansoni. Cell Immunol. 1999;195(1):37-42.

7. Shibata Y, Metzger WJ, Myrvik QN. Chitin particle-induced cellmediated immunity is inhibited by soluble mannan: mannose receptor-mediated phagocytosis initiates IL-12 production. J Immunol. 1997;159(5):2462-7.

8. Snijdewint FG, Kaliński P, Wierenga EA, Bos JD, Kapsenberg ML. Prostaglandin E2 differentially modulates cytokine secretion profiles of human T helper lymphocytes. J Immunol. 1993;150(12): 5321-9.

9. Phipps RP, Stein SH, Roper RL. A new view of prostaglandin E regulation of the immune response. Immunol Today. 1991;12 (10):349-352 .

10. Kanda N, Tani K, Enomoto U, Nakai K, Watanabe S. The skin fungus-induced Th1- and Th2-related cytokine, chemokine and prostaglandin E2 production in peripheral blood mononuclear cells from patients with atopic dermatitis and psoriasis vulgaris. Clin Exp Allergy. 2002;32(8):1243-50.

11. Blake JS, Dahl MV, Herron MJ, Nelson RD. An immunoinhibitory cell wall glycoprotein (mannan) from Trichophyton rubrum. J Invest Dermatol. 1991;96(5):657-61. 